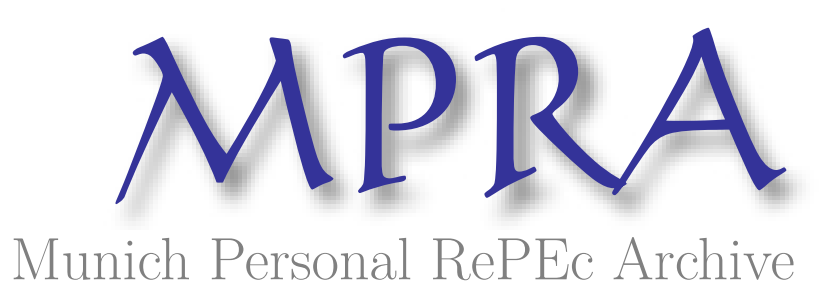

\title{
The input-output multi-dimensional analysis: theoretical framework
}

Ruiz Estrada, M.A.

University of Malaya

5 January 2010

Online at https://mpra.ub.uni-muenchen.de/40577/

MPRA Paper No. 40577, posted 08 Aug 2012 16:58 UTC 


\title{
The Input-Output Multi-Dimensional Analysis: Theoretical Framework
}

Keywords:

Econographicology, Matrix Algebra, Multi-dimensional graphs

\author{
$\underline{\text { JEL code }}$ \\ C67
}

\author{
Corresponding Author \\ Dr. Mario Arturo RUIZ Estrada, \\ Faculty of Economics and Administration \\ Department of Economics \\ University of Malaya \\ Kuala Lumpur 50603 \\ [H/P] (60) 12-6850293 \\ [E-mail] marioruiz@um.edu.my \\ [Website] www.econonographication.com
}

"I never follow old ideas...Because the ideas always keep in constant transformation..." 


\begin{abstract}
This paper is interested to propose an alternative approach entitled the input-output multi-dimensional analysis. This approach is based on the interaction among four production sectors (agriculture, light industry, heavy industry and services) by "i" number of sub-sectors under the uses of " $\mathrm{j}$ " number of commodities. The idea is to generate an alternative mathematical and graphical modeling approach to analyze the behavior of four production sectors and "i" number of sub-sectors to get the final output of the economy under the construction of the GDP-Surface.
\end{abstract}

\title{
1. Introduction
}

The modeling of the input-output models were introduced by Professor Wassily Leontief, he deals with a particular question: "What level of output should each of the $n$ industries in an economy produce, in order that it will just be sufficient to satisfy the total demand for that product..." (Leontief, 1951). The model he proposes is a static and partial equilibrium version. Hence, the input-output analysis is not showing a general equilibrium or dynamic modeling. Therefore, the main idea to generate the input-output analysis always is simplified by three production sectors (agriculture, industry and services), in the original paper wrote by Professor Leontief the services sector appear as householders (Leontief, 1985). The basic structure of the input-output table shows a large number of production sectors, but we can observe that almost all the examples are based on the uses of three sectors. This model has a serial of assumptions follow by: first assumption is that each production sector produces a single homogeneous commodity. Second assumption is that the model is working under a fixed input ratio. Third assumption is that all production sectors work under the constant returns to scale. Maybe this model looks simplistic but we can find that the great contribution of Professor Leontief is based on the economic modeling shows an alternative view, how the economy is working based on the interaction of different industries from different production sectors such as agriculture, industry and services sector and finally is to calculate the minimum of output to produce a specific commodity to satisfied the basic demand of any country.

Hence, this paper proposes the uses an alternative mathematical and graphical modeling approach to study input-output analysis from a multi-dimensional perspective. Our model is called "The Input-Output Multi-Dimensional Analysis". We try to incorporate a large number of commodities " $\mathrm{j}$ ", production sub-sectors "i $\mathrm{i}$ " and four 
production sectors in our analysis. It is based on the application of Econographicology, matrix algebra, multi-dimensional partial differentiation and economic modeling in real time.

\section{The Input-Output Multi-Dimensional Analysis}

Initially, we have a large number of " $\mathrm{j}$ " commodities generated by "i $\mathrm{i}$ " number of production sub-sectors by four production sectors (See Expression 1). In our case we have four production sectors follow by the agriculture, light industry (manufacturing), heavy industry (under the production of capital goods) and services (See Expression 3, 5, 7 and 9). These four production sectors final output depend on the final total outputs from all production sub-sectors in each of the four production factors respectively (See Expression 2, 4, 6 and 8). For example, the agriculture sector (production sector one "S1") exist the production of " $\mathrm{j}$ " number of commodities by " $\mathrm{i}$ " sub-sectors. A sub-sector into the agriculture sector or "S-1", we can mention the production of coffee by a large number of coffee plantations. Therefore, we assume that the production of any commodity by each sub-production sector is related to the fast technological challenges and the domestic and international demand in the market. In the other hand, we assume in our model that the innovation, research and development of new commodities under low cost production can generate high demand into different markets simultaneously. We can observe also that each production sub-sector can show unexpected high or low intensive exchange of commodities among all sub-sectors in the same production sector (See Expression 1), it is based on the idea that the market always keep in a dynamic imbalance state (Ruiz, 2008), to support our argument we application the assumption Omnia Mobilis (Ruiz, Yap and Shyamala, 2007.b) to generate the relaxation of the exchange of commodities among all production sectors and sub-sectors in the same production sector.

$$
\begin{aligned}
& \delta \mathrm{S}_{\mathrm{lij}}: \delta \mathrm{S}_{\mathrm{lij}} \\
& \text { Where } \quad \mathrm{i}=\{0,1,2, \ldots, \infty \ldots\} \\
& \mathrm{j}=\{0,1,2, \ldots, \infty \ldots\} \\
& 1=\{1,2,3,4\} \\
& 1=\text { Production sector } \mathrm{i}=\text { sub-sector } \mathrm{j}=\text { commodities } \text {. }
\end{aligned}
$$

The equation (1) exist two premises follow by:

a. If $\delta \mathrm{S}_{\mathrm{lij}}=\delta \mathrm{S}_{\mathrm{lij}}$ then the final output into the exchange of this sub-production sector became 0 .

b. If $\delta \mathrm{S}_{\mathrm{lij}} \neq \delta \mathrm{S}_{\mathrm{lij}}$ then the final output into the exchange of this sub-production sector became a real or negative number. 
The sector one (S-1) represent the agriculture sector, this production sector basically shows a large number of sub-sectors and each sub-sector exist the exchange of infinity number of commodities among all sub-sectors in the same production sector. The final output into the box below of each matrix can show the final output after we calculate the exchange of commodities among all sub-sectors simultaneously. The same situation is possible to be observed into the sector- 2 (light industry), sector-3 (heavy industry) and sector-4 (Services).

\section{(2) Sector S-1}

\begin{tabular}{|c|c|c|c|c|c|c|c|}
\hline Sector $S_{1}$ & $\mathrm{G}_{100}$ & $\mathrm{G}_{101}$ & $\mathrm{G}_{102}$ & .. & - & $\mathrm{G}_{10 \mathrm{j}}$ & $\mathrm{S}_{1} \mathrm{TO}$ \\
\hline $\mathrm{G}_{100}$ & 0 & {$\left[\delta \mathrm{G}_{100}: \delta \mathrm{G}_{101}\right]$} & • & • & - & {$\left[\delta \mathrm{G}_{100}: \delta \mathrm{G}_{10 \mathrm{j}}\right]$} & $\left.{ }_{\mathrm{j}}\right] \mathrm{S}_{100}$ \\
\hline $\mathrm{G}_{101}$ & {$\left[\delta \mathrm{G}_{101}: \delta \mathrm{G}_{100}\right]$} & 0 & . & . & & {$\left[\delta \mathrm{G}_{101}: \delta \mathrm{G}_{10 \mathrm{j}}\right]$} & $\left.{ }_{\mathrm{j}}\right] \mathrm{S}_{110}$ \\
\hline $\mathrm{G}_{102}$ & • & • & & $\cdots$ & & - & - \\
\hline - & - & • & & ... & & - & • \\
\hline $\mathrm{G}_{10 \mathrm{j}}$ & {$\left[\delta \mathrm{G}_{10 \mathrm{j}}: \delta \mathrm{G} 1_{00}\right]$} & $\left.{ }_{0}\right]\left[\delta \mathrm{G}_{100}: \delta \mathrm{G}_{101}\right]$ & & ‥ & & 0 & $\Sigma \mathrm{S}_{10 \mathrm{j}}$ \\
\hline $\mathrm{S}_{1} \mathrm{TI}$ & $\Sigma_{S_{100}}$ & $\Sigma_{S_{101}}$ & & & & $\Sigma_{\mathrm{G}_{10}}$ & $\mathrm{i}_{10 \mathrm{j}} \Sigma$ \\
\hline
\end{tabular}

Note: "G" represents commodities

(3) $\quad \Sigma \mathrm{S}_{1}=f\left(0 \overline{\eta \Gamma}\left[\delta \mathrm{G}_{100}: \delta \mathrm{G}_{101}\right] \bar{\eta} \cdots \bar{\eta}\left[\delta \mathrm{G}_{100}: \delta \mathrm{G}_{10 \mathrm{j}}\right] \bar{\eta} \cdots \overline{\eta \Gamma}\left[\delta \mathrm{G}_{10 \mathrm{j}}: \delta \mathrm{G}_{101}\right] \overline{\eta \Gamma} 0\right)$

\section{(4) $\underline{\text { Sector } \mathbf{S - 2}}$}

\begin{tabular}{|c|c|c|c|c|c|c|c|}
\hline Sector $S_{2}$ & $\mathrm{G}_{210}$ & $\mathrm{G}_{211}$ & $\mathrm{G}_{212}$ & - & - & $\mathrm{G}_{21 \mathrm{j}}$ & $\mathrm{S}_{2} \mathrm{TO}$ \\
\hline $\mathrm{G}_{210}$ & 0 & {$\left[\delta \mathrm{G}_{210}: \delta \mathrm{G}_{211}\right]$} & & - & • & - $\left[\delta \mathrm{G}_{210}: \delta \mathrm{G}_{21 \mathrm{j}}\right]$ & ] $\Sigma_{S}$ \\
\hline $\mathrm{G}_{211}$ & {$\left[\Delta \mathrm{g}_{211}: \delta \mathrm{G}_{210}\right]$} & 0 & ․ & 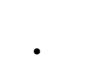 & . & {$\left[\delta \mathrm{G}_{21}: \delta \mathrm{G}_{21 \mathrm{j}}\right]$} & $\Sigma_{\mathrm{S}}$ \\
\hline $\mathrm{G}_{212}$ & - & - & & . & & - & \\
\hline - & - & - & & & . & . & • \\
\hline $\mathrm{G}_{21 \mathrm{j}}$ & {$\left[\delta \mathrm{S}_{21 \mathrm{j}}: \delta \mathrm{S}_{210}\right]$} & {$\left[\delta \mathbf{S}_{21 \mathrm{j}}: \delta \mathbf{S}_{211}\right]$} & & & & 0 & $\Sigma_{\mathrm{s}}$ \\
\hline $\mathrm{S}_{2} \mathrm{TI}$ & $\Sigma_{\mathrm{S} 2_{10}}$ & $\Sigma_{\mathrm{S} 2_{11}}$ & & & & $\Sigma_{\mathrm{S}_{21 \mathrm{j}}}$ & \\
\hline
\end{tabular}

(5) $\quad \Sigma \mathrm{S}_{2}=f\left(0 \overline{\mathrm{\Gamma}}\left[\Delta \mathrm{g}_{210}: \delta \mathrm{G}_{211}\right] \bar{\eta} \cdots \overline{\eta \Gamma}\left[\delta \mathrm{G}_{210}: \delta \mathrm{G}_{21 \mathrm{j}}\right] \bar{\eta} \cdots \overline{\eta \Gamma}\left[\delta \mathrm{G}_{21 \mathrm{j}}: \delta \mathrm{G}_{211}\right] \overline{\eta \Gamma} 0\right)$ 
(6) Sector S-3

\begin{tabular}{|c|c|c|c|c|c|c|}
\hline Sector $\mathrm{S}_{3}$ & $\mathrm{G}_{320}$ & $\mathrm{GS}_{321}$ & $\mathrm{G}_{322}$ & . . & - $\quad \mathrm{G}_{32 \mathrm{j}}$ & $\mathrm{S}_{3} \mathrm{TO}$ \\
\hline $\mathrm{G}_{320}$ & 0 & $\left.\Delta \mathrm{G}_{320}: \delta \mathrm{G}_{321}\right]$ & - & • & - $\left[\delta \mathrm{G}_{320}: \delta \mathrm{G}_{32} \mathrm{j}\right]$ & $\left.{ }_{2 \mathrm{j}}\right] \sum_{\mathrm{S}_{320}}$ \\
\hline $\mathrm{G}_{321}$ & {$\left[\delta \mathrm{G}_{321}: \delta \mathrm{G}_{320}\right]$} & 0 & • & . & {$\left[\delta \mathrm{G}_{21}: \delta \mathrm{G}_{2 \mathrm{j}}\right]$} & $\Sigma S_{321}$ \\
\hline $\mathrm{G}_{322}$ & • & . & & $\ldots$ & . & . \\
\hline • & . & . & & $\ldots$ & . & . \\
\hline $\mathrm{G}_{32 \mathrm{j}}$ & {$\left[\Delta \mathrm{g}_{32} ; \delta \mathrm{G}_{320}\right]$} & {$\left[\delta \mathrm{G}_{32} ; \delta \mathrm{G}_{32}\right.$} & & . & 0 & $\Sigma_{\mathrm{S}_{32 \mathrm{j}}}$ \\
\hline $\mathrm{G}_{3} \mathrm{TI}$ & $\Sigma_{\mathrm{S}_{320}}$ & $\Sigma_{S_{321}}$ & & $\ldots$ & $\Sigma_{S_{32 j}}$ & $\sum \Sigma_{S_{3}}$ \\
\hline
\end{tabular}

(8) Sector S-4

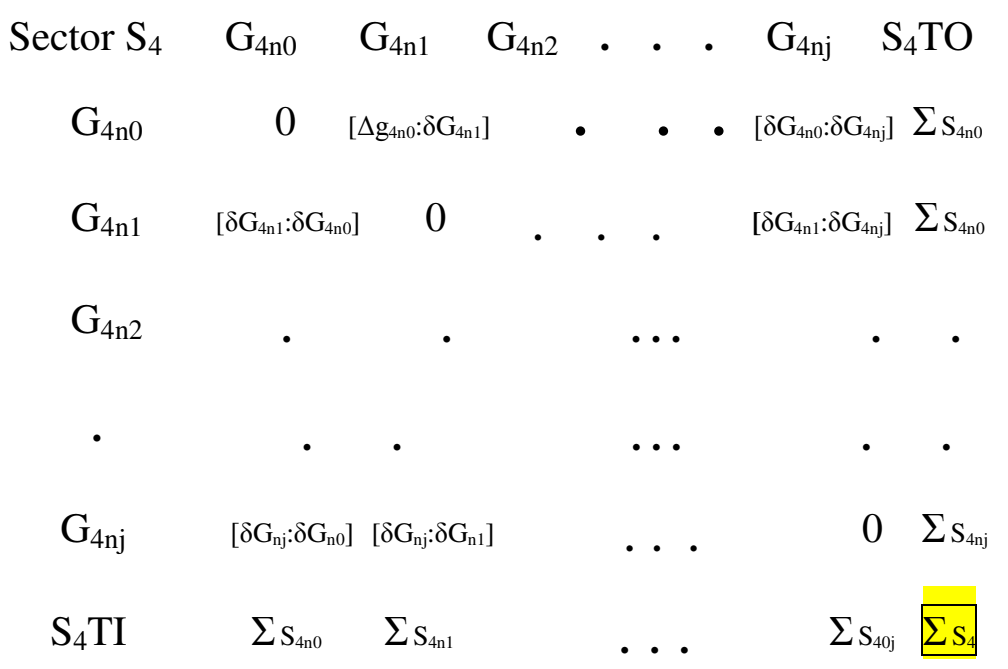

(9) $\Sigma \mathrm{S}_{4}=f\left(0 \overline{\eta \Gamma}\left[\delta \mathrm{G}_{4 \mathrm{n} 0}: \delta \mathrm{G}_{4 \mathrm{n} 1}\right] \overline{\eta \Gamma} \cdots \overline{\eta \Gamma}\left[\delta \mathrm{G}_{4 \mathrm{n} 0}: \delta \mathrm{G}_{4 \mathrm{nj}}\right] \overline{\eta \Gamma} \cdots \overline{\eta \Gamma}\left[\delta \mathrm{G}_{4 \mathrm{nj}}: \delta \mathrm{G}_{4 \mathrm{n} 1}\right] \overline{\eta \Gamma} 0\right)$

In this part of our model, we suggest to plot each production sector into four different surfaces, to build each surface, we need to use such as reference all production sub-sector outputs can be plotted on the surface mapping coordinate system, it can facilitate to build each multi-dimensional surface for each production sector. After we plot each production sub-sector, we proceed to join all production sub-sectors by strait lines from the same production sector until we are available to build a single surface. The main idea to build the four multi-dimensional surfaces is to observe the behavior of the exchange of all production sub-sectors "i" by the exchange of a large number of commodities produced by them in the same graphical space. We would like to remark 
that in the center part of each surface is equal to 0 . The reason is that the same sub-sector cannot sell and buy the same commodity by itself (See Figure 1).

Figure 1: A Multi-dimensional Surface by Production Sector

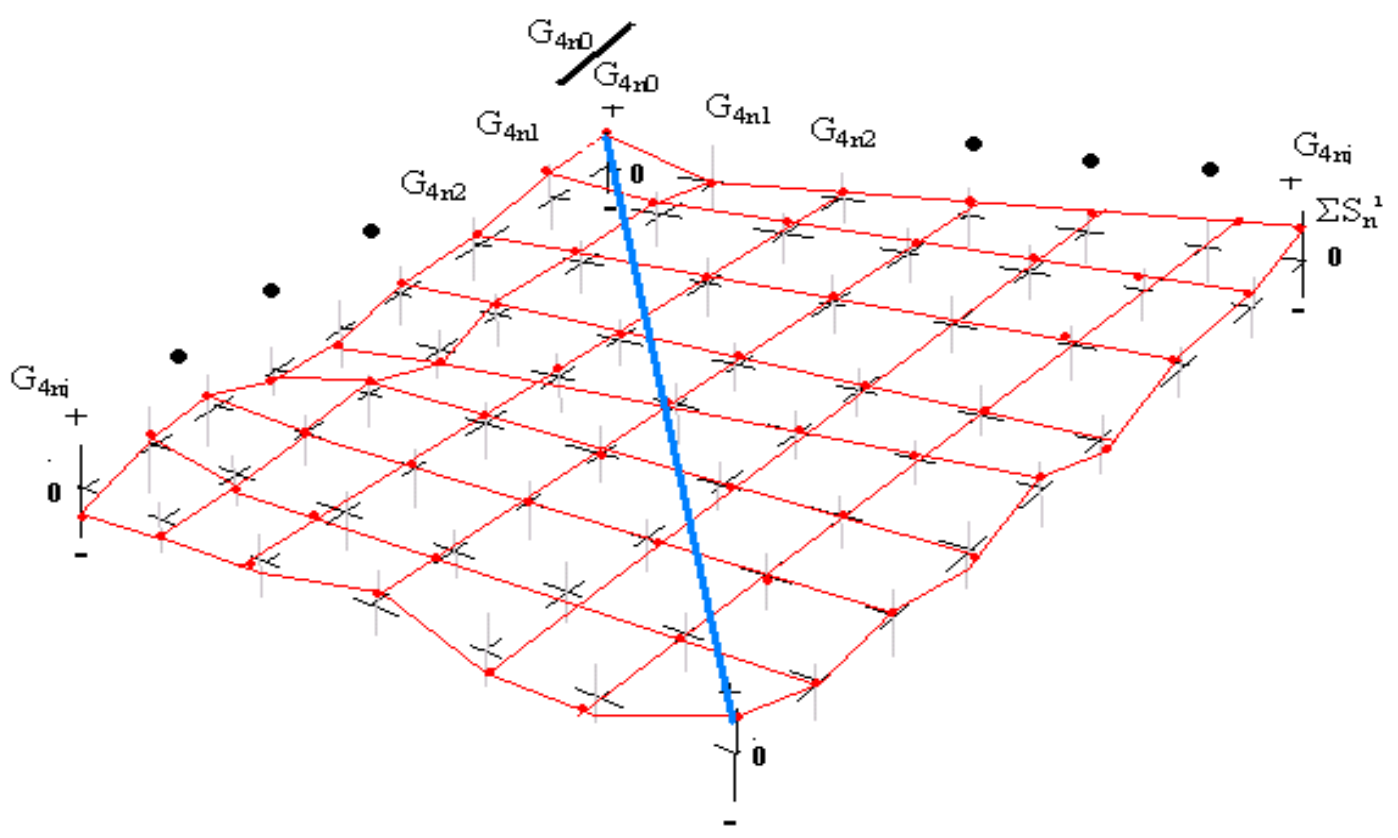

The input-output multi-dimensional analysis request the application of the multidimensional partial differentiation (See Annex) to observe the changes of two periods of time between the final time $(\mathrm{t}+1)$ and the initial time $(\mathrm{t})$. Also in this part of the model, we suggest the application of the economic modeling in real time “" (Ruiz, 2009) that consist in successive application of differentiations to observe the changes into the four production sectors simultaneously (See Expression 10). And also we suggest to apply the inter-link of all production sub-sectors based on the application of the inter-link coordinate axis condition that is represented by “ $\overline{~ " . ~}$

$$
\begin{aligned}
& \text { 次 } \Sigma \mathrm{S}_{1}{ }^{\mathrm{i}} \equiv \Sigma \mathrm{S}_{1}{ }^{\prime}=\delta f^{\prime}\left(\mathrm{S}_{1}\right)_{\mathrm{t}} / \delta\left(\mathrm{S}_{1}\right)_{\mathrm{t}+1} \mathrm{~d} \mathrm{~S}_{1} \overline{7 \Gamma} \Sigma \mathrm{S}_{1}{ }^{\prime}=\delta f^{\prime \prime}\left(\mathrm{S}_{1}\right)_{\mathrm{t}} / \delta\left(\mathrm{S}_{1}\right)_{\mathrm{t}+1} \mathrm{~d}^{2} \mathrm{~S}_{1} \overline{\eta \Gamma} \mathrm{S}_{1}{ }^{\infty}=\delta \\
& f^{\infty}\left(\mathrm{S}_{1}\right) / \delta\left(\mathrm{S}_{1}\right)_{\mathrm{t}+1} \mathrm{~d}^{\infty} \mathrm{S}_{1}
\end{aligned}
$$
$\delta\left(\mathrm{S}_{2}\right)_{\mathrm{t}+1} \mathrm{~d}^{\infty} \mathrm{S}_{2}$

$\not \mathrm{S}_{3}{ }^{\mathrm{i}} \equiv \Sigma \mathrm{S}_{3}{ }^{\prime}=\delta f^{\prime}\left(\mathrm{S}_{3}\right)_{\mathrm{t}} / \delta\left(\mathrm{S}_{3}\right)_{\mathrm{t}+1} \mathrm{~d} \mathrm{~S}_{3} \overline{7 \Gamma} \Sigma \mathrm{S}_{3}{ }^{\prime \prime}=\delta f^{\prime \prime}\left(\mathrm{S}_{3}\right)_{\mathrm{t}} / \delta\left(\mathrm{S}_{3}\right)_{\mathrm{t}+1} \mathrm{~d}^{2} \mathrm{~S}_{3} \overline{7 \Gamma} \Sigma \mathrm{S}_{3}{ }^{\infty}=\delta f^{\infty}\left(\mathrm{S}_{3}\right) /$ $\delta\left(\mathrm{S}_{3}\right)_{\mathrm{t}+1} \mathrm{~d}^{\infty} \mathrm{S}_{3}$

${ }^{\prime} \Sigma \mathrm{S}_{4}{ }^{\mathrm{i}} \equiv \Sigma \mathrm{S}_{4}{ }^{\prime}=\delta f^{\prime}\left(\mathrm{S}_{4}\right)_{\mathrm{t}} / \delta\left(\mathrm{S}_{4}\right)_{\mathrm{t}+1} \mathrm{~d}^{\prime} \mathrm{S}_{4} \overline{7 \Gamma} \Sigma \mathrm{S}_{4}{ }^{\prime \prime}=\delta f^{\prime \prime}\left(\mathrm{S}_{4}\right)_{\mathrm{t}} / \delta\left(\mathrm{S}_{4}\right)_{\mathrm{t}+1} \mathrm{~d}^{2} \mathrm{~S}_{4} \overline{\eta \Gamma} \Sigma \mathrm{S}_{4}{ }^{\infty}=\delta f^{\infty}\left(\mathrm{S}_{4}\right) /$ $\delta\left(\mathrm{S}_{4}\right)_{\mathrm{t}+1} \mathrm{~d}^{\infty} \mathrm{S}_{4}$ 
However, the construction and the final analysis of the input-output multi-dimensional analysis consist in plot all multi-dimensional partial differentiations from each production sector: agriculture, light industry, heavy industry and services (See Expression 11) on the four dimensional physical space coordinate system. (See Ruiz, 2008.a.). In fact, to join the four production sectors into the same graphical modeling, we suggest inter-link the four production sectors based on the application of the inter-link of the general coordinate condition that is represented by “卢”.

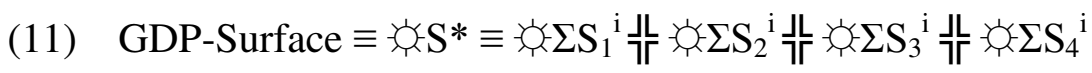

The final output of the result input-output multi-dimensional analysis, we are calling "GDP-Surface". It is depend on the final position that the GDP-surface shows into the four dimensional physical space coordinate system. We have four possible results (See Expression 12, 13, 14 and 15) to analyze the behavior of the GDP according to the speed of exchange of goods and services (economic activity) by production sector and production sub-sectors.

$$
\begin{aligned}
& \mathrm{S}^{*} \equiv \\
& \left\{\text { if }+S^{*} \cap R_{+} \text {then the surface } \equiv \text { Economic Growth Synchronized }\right\}
\end{aligned}
$$

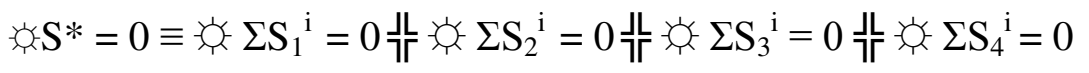

$$
\begin{aligned}
& \left\{\text { if } \not S^{*} \cap 0 \text { then the surface } \equiv \text { General Economic Stagnation }\right\}
\end{aligned}
$$

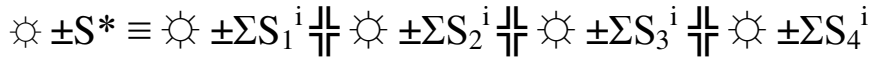

$$
\begin{aligned}
& \left\{\text { if } \mathrm{S}^{*} \cap \mathrm{R}_{+/ \text {- }} \text { then the surface } \equiv \text { Irregular Economic Growth }\right\}
\end{aligned}
$$

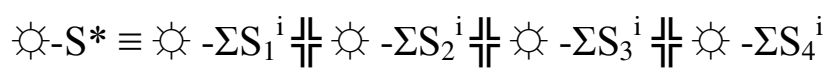

$\left\{\right.$ if $: \mathrm{S}^{*} \cap \mathrm{R}$. then the surface $\equiv$ Economic Recession $\}$ 
Figure 2: I-O Multi-dimensional Graphical Analysis

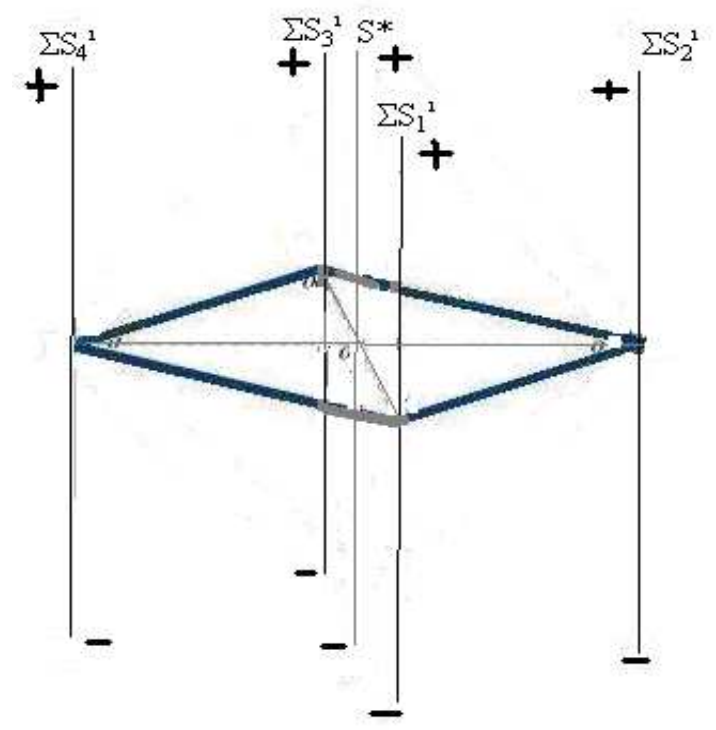

\section{Conclusion}

We can observe that the input-output multi-dimensional analysis is available to catch up the interaction of a large number of commodities exchange among different production sub-sectors in the same production sector, in the same model also is possible to observe the exchange among the four productions sectors (agriculture, light industry, heavy industry and services sector) by the application of multi-dimensional partial differentiations on complex group of functions that they are interacting in the same mathematical and graphical modeling. Finally, the contribution of Professor Leontief was great, but not enough to explain the behavior of the dynamic economy behaviors in our times.

\section{References}

Ruiz Estrada, M. A. (2007.a). "Econographicology”, International Journal of Economics Research (IJER), Vol 4-1. pp. 93-104.

Ruiz Estrada, M.A., Nagaraj, S. and Yap, S.F. (2007.b). "Beyond the Ceteris Paribus Assumption: Modeling Demand and Supply Assuming Omnia Mobilis". FEA-Working Paper No.2007-9, pp.1-15.

Ruiz Estrada, M.A., (2008). "Is the Market in Dynamic Imbalance State ?". FEAWorking Paper No.2008-4, pp.1-15. 
Ruiz Estrada, M.A., (2009). “Economic Modeling in real Time?”. FEA-Working Paper No.2009-11, pp.1-15.

Leontief, W. (1951). The Structural of America Economy 1919-1939, Second Edition Input Output Economic. Oxford University Press.

Leontief, W. (1951). The Structural of America Economy 1919-1939, Second Edition Input Output Economic. Oxford University Press.

\section{Annex}

(1) $\mathrm{dy}_{\mathrm{ij}} / \mathrm{dx}_{\mathrm{ij}}=0$ or $f^{\prime}\left(\mathrm{x}_{\mathrm{ij}}\right)=0$

(2) $\mathrm{d} / \mathrm{dx}_{\mathrm{ij}}=\mathrm{nx} \mathrm{n}_{\mathrm{ij}}^{\mathrm{n}-1}$ or $f^{\prime}\left(\mathrm{x}_{\mathrm{ij}}\right)=\mathrm{nx^{ \textrm {n } } - 1}{ }_{\mathrm{ij}}$

(3) $d / d c x_{i j}=c n x^{n-1}$ ij or $f^{\prime}\left(x_{i j}\right)=c n x^{n-1} i j$

(4) $\mathrm{d} / \mathrm{dx}_{\mathrm{ij}}\left[\alpha_{\mathrm{ij}}\left(\mathrm{x}_{\mathrm{ij}}\right) \pm \theta_{\mathrm{ij}}\left(\mathrm{x}_{\mathrm{ij}}\right) \pm \ldots \pm . \lambda_{\mathrm{ij}}\left(\mathrm{x}_{\mathrm{ij}}\right)\right]=\mathrm{d} / \mathrm{dx}_{\mathrm{ij}} \alpha\left(\mathrm{x}_{\mathrm{ij}}\right) \pm \mathrm{d} / \mathrm{d}_{\mathrm{ij}} \theta_{\mathrm{ij}}\left(\mathrm{x}_{\mathrm{ij}}\right) \pm \ldots \pm . \lambda_{\mathrm{ij}}\left(\mathrm{x}_{\mathrm{ij}}\right)$

$$
\text { or } \alpha^{\prime}\left(\mathrm{x}_{\mathrm{ij}}\right) \pm \theta^{\prime}\left(\mathrm{x}_{\mathrm{ij}}\right) \pm \ldots \pm . \lambda^{\prime}\left(\mathrm{x}_{\mathrm{ij}}\right)
$$

(5) $\mathrm{d} / \mathrm{d} \mathrm{x}_{\mathrm{ij}}\left[\alpha_{\mathrm{ij}}\left(\mathrm{x}_{\mathrm{ij}}\right) \theta_{\mathrm{ij}}\left(\mathrm{x}_{\mathrm{ij}}\right) \ldots \lambda_{\mathrm{ij}}\left(\mathrm{x}_{\mathrm{ij}}\right)\right]=\alpha\left(\mathrm{x}_{\mathrm{ij}}\right) \mathrm{d} / \mathrm{dx} \mathrm{x}_{\mathrm{ij}}+\theta_{\mathrm{ij}}\left(\mathrm{x}_{\mathrm{ij}}\right)+\ldots+. \lambda_{\mathrm{ij}}\left(\mathrm{x}_{\mathrm{ij}}\right)$

$$
\begin{aligned}
& \alpha\left(\mathrm{x}_{\mathrm{ij}}\right)+\theta_{\mathrm{ij}}\left(\mathrm{x}_{\mathrm{ij}}\right) \mathrm{d} / \mathrm{dx}_{\mathrm{ij}}+\ldots+. \lambda_{\mathrm{ij}}\left(\mathrm{x}_{\mathrm{ij}}\right) \\
& \alpha\left(\mathrm{x}_{\mathrm{ij}}\right)+\theta_{\mathrm{ij}}\left(\mathrm{x}_{\mathrm{ij}}\right)+\ldots+. \lambda_{\mathrm{ij}}\left(\mathrm{x}_{\mathrm{ij}}\right) \mathrm{d} / \mathrm{d} \mathrm{x}_{\mathrm{ij}} \ldots
\end{aligned}
$$

(6) $\mathrm{d} / \mathrm{dx} \mathrm{x}_{\mathrm{ij}}\left[\alpha_{\mathrm{ij}}\left(\mathrm{x}_{\mathrm{ij}}\right) / \theta_{\mathrm{ij}}\left(\mathrm{x}_{\mathrm{ij}}\right) \ldots \lambda_{\mathrm{ij}}\left(\mathrm{x}_{\mathrm{ij}}\right)\right]=\alpha\left(\mathrm{x}_{\mathrm{ij}}\right) \mathrm{d} / \mathrm{dx} \mathrm{x}_{\mathrm{ij}}+\theta_{\mathrm{ij}}\left(\mathrm{x}_{\mathrm{ij}}\right)+\ldots+\lambda_{\mathrm{ij}}\left(\mathrm{x}_{\mathrm{ij}}\right) /\left[\theta_{\mathrm{ij}}\left(\mathrm{x}_{\mathrm{ij}}\right)+\ldots+\lambda_{\mathrm{ij}}\left(\mathrm{x}_{\mathrm{ij}}\right)\right]^{2}$

$$
\begin{aligned}
& \mathrm{d} / \mathrm{dx}_{\mathrm{ij}}\left[\theta_{\mathrm{ij}}\left(\mathrm{x}_{\mathrm{ij}}\right) / \alpha_{\mathrm{ij}}\left(\mathrm{x}_{\mathrm{ij}}\right) \ldots \lambda_{\mathrm{ij}}\left(\mathrm{x}_{\mathrm{ij}}\right)\right]=\alpha\left(\mathrm{x}_{\mathrm{ij}}\right)+\theta_{\mathrm{ij}}\left(\mathrm{x}_{\mathrm{ij}}\right) \mathrm{d} / \mathrm{dx_{ \textrm {ij } }}+\ldots+\lambda_{\mathrm{ij}}\left(\mathrm{x}_{\mathrm{ij}}\right) /\left[\alpha_{\mathrm{ij}}\left(\mathrm{x}_{\mathrm{ij}}\right)+\ldots+\lambda_{\mathrm{ij}}\left(\mathrm{x}_{\mathrm{ij}}\right)\right]^{2} \\
& \mathrm{~d} / \mathrm{dx}_{\mathrm{ij}}\left[\lambda_{\mathrm{ij}}\left(\mathrm{x}_{\mathrm{ij}}\right) / \alpha_{\mathrm{ij}}\left(\mathrm{x}_{\mathrm{ij}}\right) \ldots \theta_{\mathrm{ij}}\left(\mathrm{x}_{\mathrm{ij}}\right)\right]=\alpha\left(\mathrm{x}_{\mathrm{ij}}\right)+\theta_{\mathrm{ij}}\left(\mathrm{x}_{\mathrm{ij}}\right)+\ldots+\lambda_{\mathrm{ij}}\left(\mathrm{x}_{\mathrm{ij}}\right) \mathrm{d} / \mathrm{d} \mathrm{x}_{\mathrm{ij}} /\left[\alpha_{\mathrm{ij}}\left(\mathrm{x}_{\mathrm{ij}}\right)+\ldots+\theta_{\mathrm{ij}}\left(\mathrm{x}_{\mathrm{ij}}\right)\right]^{2}
\end{aligned}
$$

(7) $\mathrm{d} / \mathrm{dx}_{0 \mathrm{j}}\left[\alpha_{0 \mathrm{j}}{ }^{\prime}\left(\mathrm{x}_{0 \mathrm{j}}\right) \overline{7 \Gamma} \theta_{0 \mathrm{j}}{ }^{\prime}\left(\mathrm{x}_{0 \mathrm{j}}\right) \overline{7 \Gamma} \ldots \overline{\bar{\gamma}} \lambda_{0 \mathrm{j}}{ }^{\prime}\left(\mathrm{x}_{0 \mathrm{j}}\right)\right] \ldots$

$\mathrm{d} / \mathrm{dx}_{1 \mathrm{j}}\left[\alpha_{1 \mathrm{j}}{ }^{\prime}\left(\mathrm{x}_{1 \mathrm{j}}\right) \overline{\mathrm{T}} \theta_{1 \mathrm{j}}{ }^{\prime}\left(\mathrm{x}_{1 \mathrm{j}}\right) \overline{\mathrm{T}} \ldots \overline{\mathrm{T}} \lambda_{1 \mathrm{j}}{ }^{\prime}\left(\mathrm{x}_{1 \mathrm{j}}\right)\right] \ldots$

$\mathrm{d} / \mathrm{dx} x_{\infty \mathrm{j}}\left[\alpha_{\infty \mathrm{j}}{ }^{\prime}\left(\mathrm{x}_{\infty \mathrm{j}}\right) \overline{7 \Gamma} \theta_{\infty \mathrm{j}}{ }^{\prime}\left(\mathrm{x}_{\infty \mathrm{j}}\right) \overline{7 \Gamma} \ldots \overline{\bar{\gamma}} \lambda_{\infty \mathrm{j}}{ }^{\prime}\left(\mathrm{x}_{\infty \mathrm{j}}\right)\right] \ldots$

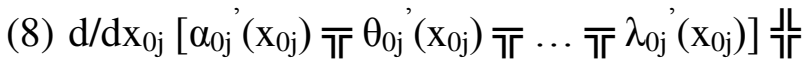

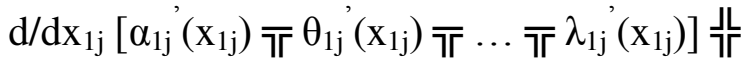

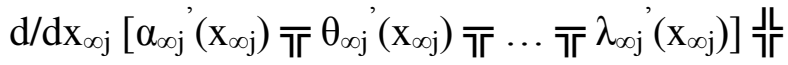

